\title{
Revisão da literatura sobre as concepções dos profissionais de saúde sobre o uso de drogas no Brasil: modelo biomédico, naturalizações e moralismos
}

| ${ }^{1}$ Pedro Henrique Antunes da Costa, ${ }^{2}$ Fernando Santana de Paiva |

Resumo: Este estudo objetivou compreender as concepções dos profissionais de saúde no Brasil sobre o uso/abuso de drogas. Trata-se de revisão da literatura nas bases de dados MEDLINE, LILACS, IBECS e Scielo. A amostra final foi composta por 22 artigos, com os resultados apontando predominância de concepções morais, naturalizantes e pautadas pelo modelo biomédico, em detrimento de perspectivas psicossociais, socioculturais ou mesmo biopsicossociais. Ao se tratar de um problema multifacetado e complexo, as tentativas de respostas devem ir numa direção de amplitude e abrangência, pensando o uso de drogas para além de uma doença meramente, ou prática necessariamente negativa, mas compreendendo os aspectos físicos, psicológicos e também sociais que o perpassam. $\mathrm{Na}$ reversão desse cenário, coloca-se a necessidade de formação/ capacitação na área, mas com reflexão sobre pressupostos e metodologias que fundamentam os processos formativos.

> Palavras-chave: transtornos relacionados ao uso de substâncias; transtornos relacionados ao uso de álcool; pessoal de saúde; recursos humanos em saúde; revisão.

\author{
1 Universidade Federal de Juiz \\ de Fora, Departamento de \\ Psicologia. Juiz de Fora-MG, \\ Brasil (phantunes.costa@ \\ gmail.com). \\ ${ }^{2}$ Universidade Federal de São \\ João Del Rei, Departamento de \\ Psicologia. São João Del Rei- \\ MG, Brasil (fernandosantana. \\ paiva@yahoo.com.br).
}


O uso de drogas existe em diferentes culturas humanas, apresentando distintas funcionalidades, cenários e padrões de consumo. Entretanto, a despeito do caráter permanente desta prática humana, ao longo do século XX vivenciou-se um cenário no qual o abuso de drogas configurou-se como um problema social e passou a exigir medidas específicas de diferentes setores sociais e governamentais. Em razão da complexidade que envolve a questão, é importante analisar o uso de drogas, bem como seus efeitos e práticas políticas decorrentes, a partir da articulação e mediação de fatores de ordem individual, sócio-cultural e políticoeconômica. Portanto, em virtude das consequências do uso nas vidas das pessoas e comunidades, bem como dos preconceitos e estigmas vigentes, acompanha-se a inserção desta temática na agenda das políticas públicas, tendo o setor saúde papel de destaque (WHITEFORD et al., 2013).

Em nosso país, também se identifica um cenário de problemas relacionados ao uso de drogas. É importante salientar, entretanto, que até meados do século XX este era um tema negligenciado pelo Estado brasileiro, não se configurando como uma política pública de caráter público-estatal. As ações governamentais impetradas adotavam um tom criminal e repressor, articulando-se ainda ao discurso médico que contribuíra para a adoção de práticas de controle e segregação dos ditos "doentes" (MACHADO; MIRANDA, 2007; ALVES, 2009).

Somente durante os anos 1970 e 1980, impulsionados por atores e movimentos sociais, com destaque para as Reformas Sanitária e Psiquiátrica, é que novos modelos de assistência aos usuários de drogas passaram a fazer parte das pautas de discussão, visando romper com a cultura da exclusão sofrida pelas pessoas com transtornos mentais e os usuários de drogas. Os novos modelos foram responsáveis por favorecer que as análises e intervenções sobre a questão do uso de drogas, seus impactos na vida dos usuários, bem como o cuidado a ser ofertado, passassem a ser responsabilidade de setores da saúde pública a partir de uma concepção biopsicossocial. De forma processual, influenciaram uma série de modificações nas políticas e leis vigentes sobre o tema, trazendo novos direcionamentos para as práticas em saúde (MACHADO; MIRANDA, 2007; ALVES, 2009).

A partir do processo em tela, o Sistema Único de Saúde (SUS) passou a ser responsável por prestar assistência aos usuários de drogas, através de serviços que devem se organizar a partir de redes. Tem-se, inclusive, em 2011, a promulgação 
da Rede de Atenção Psicossocial (RAPS), que é organizada vários níveis de atenção e complexidade para a assistência a pessoas com transtornos mentais e usuários de drogas no âmbito do SUS (BRASIL, 2011).

Nesse cenário, serviços especializados, como os Centros de Atenção Psicossocial em Álcool e Drogas (CAPSad) e leitos para desintoxicação em hospitais gerais, devem atuar de forma articulada - a partir de equipes interdisciplinares - com serviços não especializados e de base comunitária, fortalecendo as ações no âmbito da Estratégia de Saúde da Família (ESF), com Unidades Básicas de Saúde (UBS), Núcleos de Apoio à Saúde da Família (NASF), consultórios na rua, assim como outros serviços de diferentes níveis de atenção. Os dispositivos devem estar orientados pelos princípios da Atenção Primária à Saúde (APS), aumentando a gama de possibilidades para um continuum de cuidado (BRASIL, 2011), que necessita ser feito de forma compartilhada, territorializada e integral.

Contudo, apesar das recentes modificações nas formas de compreender e abordar os usuários de drogas, alguns estudos apontam que a concepção sobre o uso de drogas ainda é respaldada por modelos contrários aos preconizados pela Reforma Psiquiátrica brasileira, baseados no modelo biomédico hegemônico, que prioriza os fatores biológicos em detrimento de categorias psicossociais ou em concepções morais e preconceituosas sobre o tema (MORAES, 2008; SCHNEIDER, 2010). Entende-se, portanto, que os avanços nas legislações e políticas podem não alterar prontamente as concepções das pessoas (incluindo os profissionais) sobre o tema, sendo essas concepções hegemônicas em meio a sociedade e resultantes de processos sócio-históricos.

Em razão do entrelaçamento entre os diferentes discursos que orientam as práticas, considera-se relevante compreender as concepçōes dos profissionais de saúde sobre o uso de drogas. De acordo com o Dicionário Básico de Filosofia (JAPIASSÚ; MARCONDES, 2001), concepção seria a “[o]peração pela qual o sujeito forma, a partir de uma experiência física, moral, psicológica ou social, a representação de um objetivo de pensamento ou conceito". Portanto, considerase que essas concepções são permeadas por uma série de questôes individuais, institucionais e sócio-políticas que influenciam diretamente na maneira como os profissionais percebem e abordam o problema, impactando nas ações que serão realizadas e, consequentemente, na vida dos sujeitos que necessitam de cuidados relacionados ao uso de drogas (RONZANI; NOTO; SILVEIRA, 2014). 
Nesta perspectiva, considerando ainda as recentes modificações nas políticas de assistência aos usuários de drogas no Brasil, justifica-se a relevância de estudos que visem demonstrar um panorama sobre as concepçōes dos profissionais de saúde, sem restrições quanto a categorias profissionais, serviços e tipos de substâncias. Ressalta-se a inexistência de estudos anteriores que sintetizam o conhecimento sobre o tema, a despeito de sua pertinência. Assim, o presente artigo tem por objetivo compreender as concepçōes dos profissionais de saúde no Brasil em relação ao uso de drogas, a partir de uma revisão da literatura.

\section{Método}

Trata-se de uma revisão da literatura, com periodicidade de busca de maio a junho de 2015. As bases de dados utilizadas foram MEDLINE, LILACS, IBECS e Scielo, todas da Biblioteca Virtual de Saúde (BVS). Como forma de sistematizar o processo de busca obtendo maior controle, foi utilizado o dicionário de indexação de termos DeCS (Descritores em Ciências da Saúde) como método de definição dos descritores utilizados.

Para a busca dos artigos, os descritores transtornos relacionados ao uso de substâncias, transtornos relacionados ao uso de álcool e alcoolismo foram cruzados, a partir do operador booleano $A N D$, com pessoal de saúde e recursos humanos em saúde. Inicialmente, foram encontrados 4.886 estudos nas bases de dados selecionadas que faziam referência à associação dos termos empregados. Foram lidos todos os resumos desses trabalhos, que foram avaliados para inclusão ou exclusão na presente revisão.

Foram estabelecidos os seguintes critérios de inclusão: 1) artigos provenientes de pesquisas empíricas; 2) pesquisas que abarcassem as concepçōes dos profissionais de saúde sobre o uso de drogas; 3) estudos realizados no Brasil; 4) pesquisas com resumo e artigo completo disponíveis; e 5) trabalhos em português, inglês e espanhol. Foram excluídos os artigos com pelo menos uma das seguintes características: 1) artigos teóricos, relatos de experiências, materiais didáticos, teses, dissertaçôes; 2) pesquisas de construção e validação de instrumentos e estudos compreendendo aspectos relacionados à assistência aos usuários de drogas (tratamento, prevenção etc.) e não, necessariamente, as concepções dos profissionais de saúde sobre o uso de drogas; 3) pesquisas realizadas fora do Brasil, com graduandos ou profissionais de outros setores (educação, assistência social etc.); 4) artigos sem resumo ou 
texto completo disponíveis; 5) estudos que não estivessem em português, inglês e espanhol. Não foi especificado nenhum recorte temporal.

Após a leitura pormenorizada dos resumos, aplicação dos critérios de inclusão e exclusão e descarte dos resumos duplicados, restaram 31 trabalhos. Todos os artigos foram lidos na íntegra, sendo descartados mais 18 estudos que também não estavam de acordo com os critérios supracitados. Na leitura das referências dos 13 artigos restantes, foram identificados mais três estudos que preenchiam os critérios de inclusão, mas que não haviam sidos abarcados pelo processo de busca. Por fim, depois de consultoria e sugestôes de especialistas na área, outros seis artigos que escaparam da busca foram incorporados. Assim, a amostra final da presente revisão é composta por 22 artigos, com o processo de busca ilustrado na figura 1 .

Figura 1. Processo de busca e coleta dos artigos

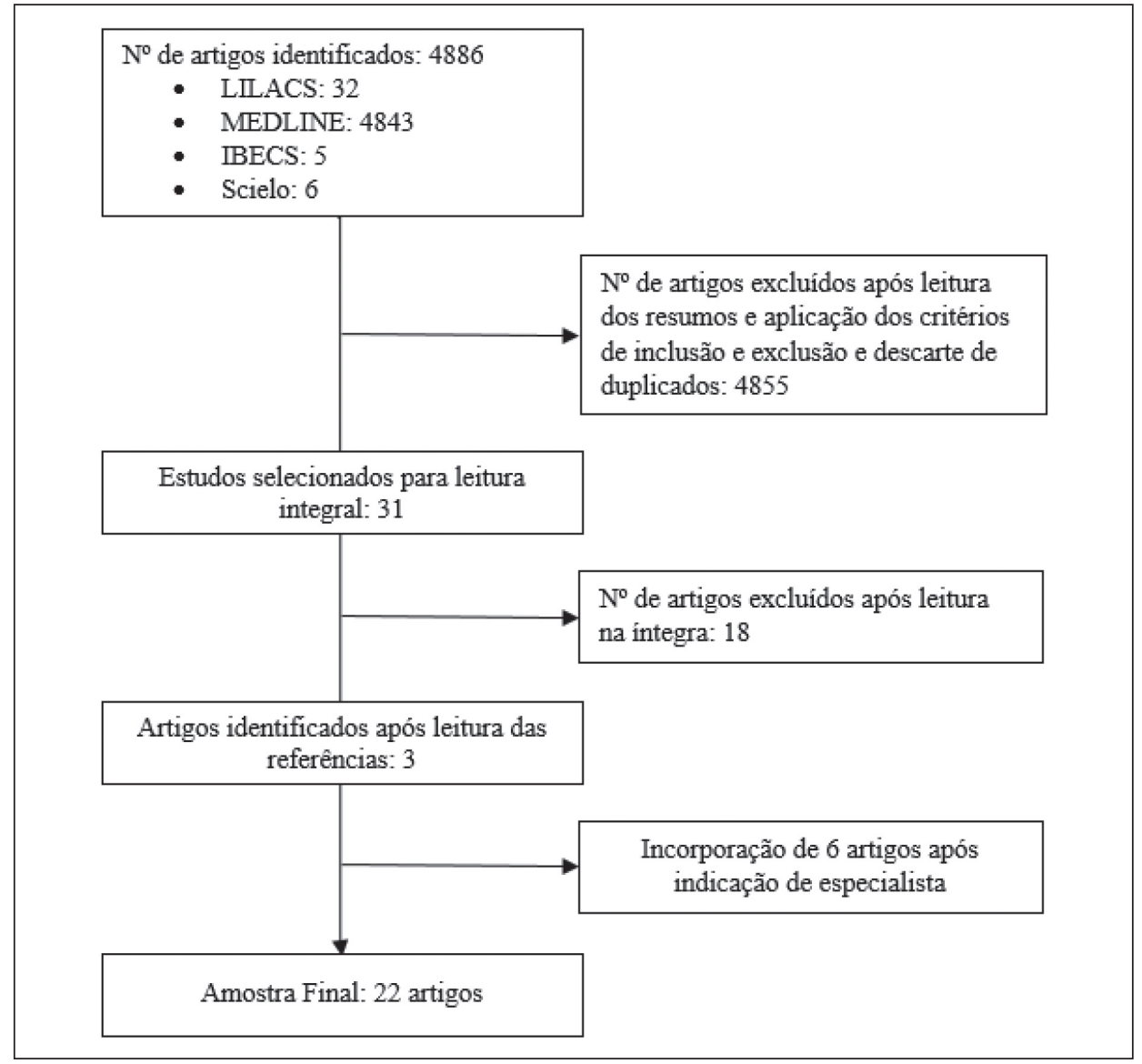

Fonte: elaboração própria 
Todo o procedimento de seleção foi feito através da avaliação por pares, com dois pesquisadores lendo os resumos e artigos e avaliando-os quanto a sua inclusão ou não na revisão, chegando a consensos. O programa EndNote web foi utilizado como ferramenta de apoio e organização do material.

Os artigos selecionados foram tabulados, com discriminação dos seguintes itens: autores, ano, país e periódico de publicação, descrição do tema de estudo, método (natureza dos estudos, participantes e instrumentos) e resultados encontrados. Por fim, realizou-se análise descritiva dos indicadores bibliométricos da amostra e qualitativa dos resultados dos artigos. Para a análise qualitativa, realizou-se análise de conteúdo do tipo temática (BARDIN, 2010), a partir dos seguintes procedimentos: 1) pré-análise, com a leitura flutuante e familiarização com o material; 2) exploração do material, extraindo categorias a partir do texto; 3) tratamento dos resultados, interpretando as categorias, sintetizando o que representavam e transformando os dados soltos em resultados brutos (BARDIN, 2010).

\section{Resultados}

\section{Indicadores bibliométricos}

Conforme mencionado, a presente revisão é composta por 22 artigos, que estão descritos, em conjunto com seus anos de publicação e objetivos, no quadro 1. Os anos de publicação variaram de 2003 a 2014, sendo 2010 o de maior número de publicações (quatro), seguido de 2006, 2008, 2009 e 2014 (três cada), 2007 (dois), 2003, 2004, 2011, 2013 (um cada). Em 2005 e 2012, nenhuma publicação foi encontrada a partir do processo de busca delineado.

\section{Quadiro 1. Síntese dos artigos que compõem a revisão}

\begin{tabular}{|l|l|l|c|c|c|c|}
\hline \multirow{2}{*}{ Autores } & Ano & Participantes & \multirow{2}{*}{$\begin{array}{c}\text { Técnicas } \\
\text { empregadas }\end{array}$} & $\begin{array}{c}\text { Modelo } \\
\text { Biomédico }\end{array}$ & $\begin{array}{c}\text { Moralismos } \\
\text { e Estigmas }\end{array}$ & Naturalização \\
\hline \multirow{2}{*}{$\begin{array}{l}\text { CASTANHA; } \\
\text { ARAÚJO }\end{array}$} & 2006 & $\begin{array}{c}\text { Entrevistas } \\
\text { Semiestruturadas, } \\
\text { Teste de } \\
\text { Comunitários de } \\
\text { Saúde (ACS) }\end{array}$ & $\begin{array}{c}\text { Associação Livre } \\
\text { de Palavras } \\
\text { (TALP) }\end{array}$ & & & X \\
\hline
\end{tabular}

continua... 


\begin{tabular}{|c|c|c|c|c|c|c|}
\hline \multirow[b]{2}{*}{ Autores } & \multirow[b]{2}{*}{ Ano } & \multirow[b]{2}{*}{ Participantes } & \multirow{2}{*}{$\begin{array}{c}\text { Técnicas } \\
\text { empregadas }\end{array}$} & \multicolumn{3}{|c|}{ Resultados } \\
\hline & & & & $\begin{array}{c}\text { Modelo } \\
\text { Biomédico }\end{array}$ & $\begin{array}{c}\text { Moralismos } \\
\text { e Estigmas }\end{array}$ & Naturalização \\
\hline GOMIDE et al. & 2010 & $\begin{array}{c}183 \text { profissionais } \\
\text { da saúde de } \\
\text { serviços em } \\
\text { saúde mental e } \\
\text { hospitais gerais }\end{array}$ & $\begin{array}{c}\text { Questionário } \\
\text { Sociodemográfico } \\
\text { e Escala de } \\
\text { estereótipos }\end{array}$ & & X & \\
\hline LIMA et al. & 2007 & $\begin{array}{l}22 \text { profissionais } \\
\text { de saúde de } \\
\text { serviços em } \\
\text { DST/AIDS }\end{array}$ & $\begin{array}{c}\text { Entrevistas } \\
\text { semiestruturadas }\end{array}$ & $\mathrm{X}$ & $\mathrm{X}$ & $\mathrm{X}$ \\
\hline MORAES & 2008 & $\begin{array}{c}36 \text { pessoas } \\
\text { (usuários, } \\
\text { acompanhantes } \\
\text { e profissionais } \\
\text { de diferentes } \\
\text { setores) }\end{array}$ & $\begin{array}{c}\text { Grupos focais, } \\
\text { observação } \\
\text { participante } \\
\text { e pesquisa } \\
\text { documental }\end{array}$ & $\mathrm{X}$ & $\mathrm{X}$ & \\
\hline $\begin{array}{l}\text { MORETTI- } \\
\text { PIRES; LIMA; } \\
\text { KATSURAYAMA }\end{array}$ & 2010 & $\begin{array}{l}\text { Seis médicos } \\
\text { da Estratégia } \\
\text { de Saúde da } \\
\text { Família (ESF) }\end{array}$ & Grupos Focais & $\mathrm{X}$ & & $\mathrm{X}$ \\
\hline $\begin{array}{l}\text { MORETTI- } \\
\text { PIRES et al. }\end{array}$ & 2011 & $\begin{array}{c}12 \text { enfermeiros } \\
\text { de ESF }\end{array}$ & $\begin{array}{c}\text { Grupos Focais } \\
\text { e entrevistas } \\
\text { semiestruturadas }\end{array}$ & $\mathrm{X}$ & $\mathrm{X}$ & $\mathrm{X}$ \\
\hline $\begin{array}{l}\text { OLIVEIRA; } \\
\text { PAIVA; } \\
\text { VALENTE }\end{array}$ & 2006 & $\begin{array}{c}19 \text { profissionais } \\
\text { de serviço } \\
\text { especializado } \\
\text { a usuários de } \\
\text { drogas }\end{array}$ & $\begin{array}{c}\text { Observação } \\
\text { de campo e } \\
\text { entrevistas } \\
\text { semiestruturadas }\end{array}$ & $\mathrm{X}$ & $\mathrm{X}$ & $\mathrm{X}$ \\
\hline PRATES et al. & 2014 & $\begin{array}{l}14 \text { enfermeiros } \\
\text { de prontos- } \\
\text { socorros }\end{array}$ & $\begin{array}{c}\text { Entrevistas } \\
\text { semiestruturadas }\end{array}$ & $\mathrm{X}$ & $\mathrm{X}$ & $\mathrm{X}$ \\
\hline REZENDE & 2003 & $\begin{array}{l}22 \text { profissionais } \\
\text { da saúde mental }\end{array}$ & Entrevistas & $\mathrm{X}$ & & $\mathrm{X}$ \\
\hline
\end{tabular}

continua... 


\begin{tabular}{|c|c|c|c|c|c|c|}
\hline \multirow[b]{2}{*}{ Autores } & \multirow[b]{2}{*}{ Ano } & \multirow[b]{2}{*}{ Participantes } & \multirow{2}{*}{$\begin{array}{c}\text { Técnicas } \\
\text { empregadas }\end{array}$} & \multicolumn{3}{|c|}{ Resultados } \\
\hline & & & & $\begin{array}{c}\text { Modelo } \\
\text { Biomédico }\end{array}$ & $\begin{array}{c}\text { Moralismos } \\
\text { e Estigmas }\end{array}$ & Naturalização \\
\hline $\begin{array}{l}\text { RONZANI; } \\
\text { HIGGINS- } \\
\text { BIDDLE; } \\
\text { FURTADO }\end{array}$ & 2009 & $\begin{array}{c}604 \\
\text { profissionais } \\
\text { da Atenção } \\
\text { Primária à Saúde } \\
\text { (APS) }\end{array}$ & $\begin{array}{c}\text { Questionários } \\
\text { sociodemográfico, } \\
\text { de julgamento } \\
\text { sobre alcoolismo, } \\
\text { problemas } \\
\text { de saúde e } \\
\text { dificuldade } \\
\text { pessoal }\end{array}$ & & $\mathrm{X}$ & \\
\hline VARGAS; LUIS & 2008 & $\begin{array}{c}10 \text { enfermeiros } \\
\text { da APS }\end{array}$ & $\begin{array}{c}\text { Entrevistas } \\
\text { semiestruturadas }\end{array}$ & $\mathrm{X}$ & X & $\mathrm{X}$ \\
\hline $\begin{array}{l}\text { BARROS; } \\
\text { PILLON }\end{array}$ & 2007 & $\begin{array}{c}286 \text { profissionais } \\
\text { de ESF }\end{array}$ & $\begin{array}{c}\text { Questionário } \\
\text { sociodemográfico } \\
\text { e escala de } \\
\text { atitudes }\end{array}$ & $\mathrm{X}$ & $\mathrm{X}$ & \\
\hline MARTINS et al. & 2009 & $\begin{array}{c}40 \text { enfermeiros } \\
\text { de Hospital } \\
\text { Geral }\end{array}$ & $\begin{array}{c}\text { Entrevistas } \\
\text { semiestruturadas }\end{array}$ & & & $\mathrm{X}$ \\
\hline PAIVA et al. & 2014 & $\begin{array}{c}10 \text { profissionais } \\
\text { da APS e Centro } \\
\text { de Referência } \\
\text { da Assistência } \\
\text { Social }\end{array}$ & $\begin{array}{c}\text { Entrevistas } \\
\text { semiestruturadas }\end{array}$ & $\mathrm{X}$ & $\mathrm{X}$ & $\mathrm{X}$ \\
\hline SCHNEIDER & 2010 & $\begin{array}{l}\text { Profissionais } \\
\text { de } 20 \text { serviços } \\
\text { de atenção a } \\
\text { usuários de } \\
\text { drogas }\end{array}$ & $\begin{array}{c}\text { Entrevistas } \\
\text { semiestruturadas }\end{array}$ & $\mathrm{X}$ & $\mathrm{X}$ & $\mathrm{X}$ \\
\hline $\begin{array}{l}\text { SOARES; } \\
\text { VARGAS; } \\
\text { FORMIGONI }\end{array}$ & 2013 & $\begin{array}{l}280 \text { enfermeiros } \\
\text { de um hospital } \\
\text { geral, APS e } \\
\text { outros serviços } \\
\text { de saúde }\end{array}$ & $\begin{array}{c}\text { Escala de } \\
\text { Atitudes } \\
\text { ao Álcool, } \\
\text { Alcoolismo e } \\
\text { Alcoolista e } \\
\text { questionário de } \\
\text { conhecimentos }\end{array}$ & & X & \\
\hline
\end{tabular}

continua... 


\begin{tabular}{|c|c|c|c|c|c|c|}
\hline \multirow[b]{2}{*}{ Autores } & \multirow[b]{2}{*}{ Ano } & \multirow[b]{2}{*}{ Participantes } & \multirow{2}{*}{$\begin{array}{l}\text { Técnicas } \\
\text { empregadas }\end{array}$} & \multicolumn{3}{|c|}{ Resultados } \\
\hline & & & & $\begin{array}{c}\text { Modelo } \\
\text { Biomédico }\end{array}$ & $\begin{array}{l}\text { Moralismos } \\
\text { e Estigmas }\end{array}$ & Naturalização \\
\hline $\begin{array}{l}\text { VARGAS; } \\
\text { LABATE }\end{array}$ & 2006 & $\begin{array}{l}196 \text { enfermeiros } \\
\text { de hospital geral }\end{array}$ & $\begin{array}{l}\text { Escala de } \\
\text { Atitudes } \\
\text { ao Álcool, } \\
\text { Alcoolismo e } \\
\text { Alcoolista }\end{array}$ & & $\mathrm{X}$ & \\
\hline $\begin{array}{l}\text { MOUTINHO; } \\
\text { LOPES }\end{array}$ & 2008 & $\begin{array}{c}18 \text { enfermeiros } \\
\text { de ESF }\end{array}$ & $\begin{array}{c}\text { Entrevistas } \\
\text { semiestruturadas }\end{array}$ & $\mathrm{X}$ & $\mathrm{X}$ & \\
\hline $\begin{array}{l}\text { SPRICIGO; } \\
\text { ALENCASTRE }\end{array}$ & 2004 & $\begin{array}{c}\text { Sete enfermeiros } \\
\text { da APS }\end{array}$ & $\begin{array}{c}\text { Entrevistas } \\
\text { semiestruturadas }\end{array}$ & $\mathrm{X}$ & $\mathrm{X}$ & \\
\hline $\begin{array}{l}\text { SILVEIRA; } \\
\text { MARTINS; } \\
\text { RONZANI }\end{array}$ & 2009 & 197 ACS & $\begin{array}{c}\text { Questionário } \\
\text { sociodemográfico } \\
\text { e Escala de } \\
\text { estereótipos }\end{array}$ & & $\mathrm{X}$ & \\
\hline SILVA; PERES & 2014 & $\begin{array}{c}12 \text { profissionais } \\
\text { da ESF }\end{array}$ & $\begin{array}{c}\text { Entrevistas } \\
\text { semiestruturadas }\end{array}$ & & $\mathrm{X}$ & $\mathrm{X}$ \\
\hline $\begin{array}{l}\text { OLIVEIRA; } \\
\text { MCCALLUM; } \\
\text { COSTA }\end{array}$ & 2010 & $22 \mathrm{ACS}$ & $\begin{array}{c}\text { Entrevistas } \\
\text { semiestruturadas, } \\
\text { observação } \\
\text { participante e } \\
\text { TALP }\end{array}$ & $\mathrm{X}$ & $\mathrm{X}$ & $\mathrm{X}$ \\
\hline
\end{tabular}

Fonte: elaboração própria.

Observou-se uma concentração de publicação em periódicos da enfermagem, com presença também de revistas multidisciplinares de saúde em geral (Saúde Pública e Coletiva) e psicologia. A maioria dos estudos era de natureza qualitativa (15 trabalhos), com um estudo de abordagem mista (quali-quanti) e seis quantitativos. Os delineamentos variaram, mas com predomínio de estudos exploratórios, de corte transversal. Os principais métodos de coleta de dados foram: entrevistas semiestruturadas (14 pesquisas); escalas mensurando atitudes, estereótipos e moralismos dos profissionais, em seis estudos; questionários sociodemográficos em quatro; grupos focais em três; observação de campo em três. Dez pesquisas utilizaram mais de uma forma de coleta dos dados.

Ao todo, 13 estudos tiveram dentre seus objetivos compreender as concepções dos profissionais de saúde sobre o uso das drogas em geral, enquanto nove focaram 
no uso de álcool especificamente. Quanto às categorias profissionais, oito estudos englobaram as concepções de enfermeiros, três de agentes comunitários de saúde (ACS) e um de médicos, sendo que o restante (10 pesquisas) abarcou equipes multidisciplinares.

Doze trabalhos se ocuparam dos profissionais da APS, sendo eles de UBS e equipes de ESF; quatro englobaram, em sua amostra, profissionais de hospitais gerais; enquanto serviços especializados em saúde mental estiveram presentes em dois artigos. CAPSad, prontos-socorros, unidade de saúde especializada na assistência a pessoas usuárias de drogas, ambulatório especializado em DST/ Aids, Centro de Referência DST/Aids, grupos de ajuda mútua, comunidades terapêuticas e programas de redução de danos estiveram representados em um estudo cada. Três estudos abarcaram em sua amostra profissionais de mais de um tipo de serviço e um artigo não especificou o local de trabalho de seus participantes.

Os estudos concentraram-se em municípios de estados do Sudeste (São Paulo, Minas Gerais e Rio de Janeiro), com 13 pesquisas nessa região. Quatro estudos foram realizados em municípios do Nordeste (Pernambuco e Bahia), dois na Norte (Amazonas) e um na região Sul (Santa Catarina). Um trabalho englobou profissionais de diversas regiōes, sem especificá-las.

\section{Síntese das principais conclusões dos}

\section{artigos selecionados: o que pensam os profissionais?}

Com relação à análise de conteúdo dos resultados dos artigos, referentes às concepçôes dos profissionais de saúde, três categorias emergiram, a saber: 1) modelo biomédico de compreensão do uso de drogas; 2) concepçôes morais e estigmatizantes; e 3) naturalização do uso e usuário de drogas. Cabe ressaltar que, apesar de descritas enquanto categorias distintas, em grande parte, elas possuem relações entre si.

\section{Modelo biomédico de compreensão do uso de drogas}

Sobre a primeira categoria observou-se que, para os profissionais de saúde, o uso de álcool e outras drogas é um problema de saúde pública, com demonstrações de preocupação sobre os potenciais prejuízos para a saúde das pessoas. Entretanto, desconsideram-se as possíveis interfaces entre os aspectos pessoais (fatores biológicos e psicológicos/subjetivos) e os determinantes sociais nas relaçoos 
estabelecidas entre as pessoas e as drogas (LIMA et al., 2007). O uso de drogas é visto majoritariamente como uma patologia (SPRICIGO; ALENCASTRE, 2004; MOUTINHO; LOPES, 2008; VARGAS; LUÍS, 2008; SCHNEIDER, 2010; PRATES et al., 2014), não identificando os diferentes padrões de uso (REZENDE, 2003; MORETTI-PIRES et al. 2011), e com grande parte dos usuários - senão todos - identificados como dependentes (PRATES et al., 2014; REZENDE, 2003). Ademais, esse uso aparece também como uma doença sem cura (MORAES, 2008), com ênfase exclusiva nos aspectos biológicos ou sobrepondo-os em detrimento das questōes sociais e psicológicas (BARROS; PILLON 2007; LIMA et al., 2007; SCHNEIDER, 2010; PRATES et al., 2014).

O foco da atuação dos profissionais é, prioritariamente, o tratamento embasado pelo modelo biomédico (BARROS; PILLON 2007; MOUTINHO; LOPES, 2008; VARGAS; LUÍS, 2008; MORETTI-PIRES et al., 2011), desconsiderando possibilidades de prevenção e promoção de saúde, ou relegando-as a outros setores, como a segurança pública (MOUTINHO; LOPES, 2008). Nessa perspectiva, a medicalização aparece enquanto forma de tratamento e a abstinência como objetivo ideal para todos os casos (REZENDE, 2003; MORAES, 2008; SCHNEIDER, 2010). Observa-se, contudo, um cenário paradoxal, onde apesar de a abstinência ser necessária para a reintegração do usuário à sociedade, caracterizando-se como uma tentativa de purificação ou salvação, o usuário ainda é percebido como um doente. Portanto, mesmo aquele abstinente estará, na melhor das hipóteses, em uma condição estável (MORAES, 2008).

Assim, os determinantes sociais nessa equação, quando aparecem, estão majoritariamente relacionados à família, numa perspectiva de superresponsabilização (PAIVA et al., 2014). Isso corre em detrimento de parcas tentativas de compreensão dos efeitos estruturais ou sócio-políticos relacionados ao problema das drogas, como: desigualdade social, desemprego, nível socioeconômico, dentre outros (REZENDE, 2003; SPRICIGO; ALENCASTRE, 2004; LIMA et al., 2007; OLIVEIRA, McCALLUM; COSTA, 2010; PAIVA et al., 2014).

Em alguns casos, como no estudo com médicos da ESF do Amazonas, houve uma aproximação com os conceitos de uso de álcool da Organização Mundial da Saúde, sob uma perspectiva ampliada de saúde (biopsicossocial). Entretanto, esses mesmos profissionais demonstram não saber lidar com os problemas relacionados 
ao uso dessa substância, inclusive com a utilização de termos estigmatizantes (MORETTI-PIRES; LIMA; KATSURAYAMA, 2010).

Como contraponto a estas perspectivas está a pesquisa de Oliveira, Paiva e Valente (2006) com profissionais de uma unidade de saúde especializada na assistência a pessoas usuárias de drogas e a seus familiares de Salvador-BA. Para os entrevistados, o uso de drogas é atravessado pelo contexto social e cultural em que se insere, com os problemas da vida, sejam eles do âmbito social, de saúde, pessoal etc., influenciando na relação que se estabelece com as drogas. Assim, esse uso não seria um "caminho sem volta”, mas um processo que é modificado de pessoa para pessoa e que depende justamente da relação estabelecida entre essas pessoas, as drogas e o lugar que estas ocupam em suas vidas. Um fator a se considerar na compreensão dos achados desse estudo é o forte embasamento na redução de danos dos profissionais, inclusive, com a presença de redutores de danos que, no caso, eram usuários e ex-usuários, entre os entrevistados. Contudo, ao enfocarem a perspectiva de gênero, os autores observaram uma série de estigmatizações e estereótipos de submissão das mulheres (OLIVEIRA et al., 2006).

\section{Concep̧̧ões morais e estigmatizantes}

Quanto à visão moral dos profissionais, as seguintes concepções sobre o uso e o usuário de drogas foram identificadas: os alcoolistas não possuem força de vontade e determinação, sendo moralmente fracos, pecadores, vagabundos, delinquentes ou na "desordem" (SPRICIGO; ALENCASTRE, 2004; OLIVEIRA et al., 2006; VARGAS; LUÍS, 2008; GOMIDE et al., 2010); devido à sua doença (dependência), vista de maneira negativa (MORAES, 2008; SOARES; VARGAS; FORMIGONI, 2013), os usuários não possuem domínio sobre suas ações ou chance de escolha, negando que estão doentes (MORETTIPIRES et al., 2011; PRATES et al., 2014), não querendo parar de beber (GOMIDE et al., 2010) ou dificultando e tumultuando o tratamento (LIMA et al., 2007; SILVEIRA; MARTINS; RONZANI, 2009; SILVA; PERES, 2014); o uso de drogas é encarado como algo sempre errado e ruim (LIMA et al., 2007), tornando o indivíduo débil e louco (VARGAS; LABATE, 2006) e associado a práticas antissociais de violência e criminalidade (MOUTINHO; LOPES, 2008; OLIVEIRA et al., 2010).

Dessa forma, observa-se outro paradoxo: apesar de ser considerada uma doença (vide categoria anterior), o uso de drogas pode ser também apontado 
como uma "escolha" do usuário, analisada pelos profissionais a partir de concepções estritamente morais (SCHNEIDER, 2010; PAIVA et al., 2014). É atribuída ao usuário a responsabilidade por sua doença (SOARES et al., 2013) e, em alguns casos, até mesmo pelo problema da violência urbana (MOUTINHO; LOPES, 2008).

Com relação às pesquisas que consideraram em sua análise as diferenças entre categorias e níveis profissionais, observou-se que os profissionais com menor nível de escolaridade apresentaram maior estereotipização e moralização ao uso e usuários de álcool em hospitais gerais e serviços de saúde mental (GOMIDE et al., 2010) e na ESF (SILVEIRA et al., 2009; RONZANI; HIGGINS-BIDDLE; FURTADO, 2009). Entretanto, Barros e Pillon (2007), ao constatarem atitudes positivas dos profissionais de ESF, identificaram que aqueles com curso superior tinham maior amplitude de conhecimento sobre os problemas físicos relacionados ao uso de drogas, mas um prognóstico não muito positivo dos usuários, enquanto os profissionais sem graduação tinham sentimentos e atitudes de maior aceitação, porém com dificuldades na abordagem à questão.

\section{Naturalização do uso e usuário de drogas}

A partir do exposto, as concepçóes encontradas pelas investigações apontam para uma naturalização do uso e usuário de drogas, onde além de não discriminados os tipos de drogas e formas de uso (MORETTI-PIRES et al., 2011), os usuários são tratados de maneira cristalizada. Ocorre, então, um processo de homogeneização das análises, intervençôes e, consequentemente, dos usuários, desconsiderando suas singularidades e necessidades (REZENDE, 2003; PAIVA et al., 2014), bem como o contexto sócio-histórico que os permeia (SCHNEIDER, 2010), aspecto esse que impacta indubitavelmente nos tratamentos ofertados pelos diferentes dispositivos das políticas públicas (SILVA; PERES, 2014).

Os estudos apontaram que os profissionais consideram como principais causas para o uso de álcool e outras drogas, os seguintes fatores: 1) curiosidade (OLIVEIRA et al., 2006) e busca de prazeres, numa perspectiva hedonista (LIMA et al., 2007; PRATES et al., 2014) ou pela insuficiência de fontes de prazer (MORETTI-PIRES et al., 2010); 2) alívio do sofrimento (PRATES et al., 2014); 3) características da personalidade e/ou transtornos mentais (REZENDE, 2003); 4) experiências pessoais e afetivas (LIMA et al., 2007); 5) fatores genéticos e hereditariedade; 6) problemas familiares (REZENDE, 2003; OLIVEIRA et 
al., 2010; MORETTI-PIRES et al., 2011); 7) possibilidade de geração de renda através da inserção nas redes de tráfico (OLIVEIRA et al., 2006).

Com relação às principais consequências do uso de drogas, foram apontados pelos profissionais: a) morte (CASTANHO; ARAÚJO, 2006; VARGAS; LUÍS, 2008); b) problemas familiares (CASTANHO; ARAÚJO, 2006; MARTINS et al., 2009; OLIVEIRA et al., 2010; MORETTI-PIRES et al., 2011); c) dificuldades gerais com a sociedade provenientes das implicaçōes financeiras ou pela natureza emocional da questão (MORETTI-PIRES et al., 2011); e d) violência e criminalidade (OLIVEIRA et al., 2010).

\section{Discussão}

Ao objetivar compreender as concepções dos profissionais de saúde sobre o uso de drogas, o presente trabalho não pretende culpabilizar esses profissionais em decorrência de suas concepções sobre o tema, nem radicalizar visões e perspectivas de atuação frente ao uso e usuário de drogas. Devido à própria amplitude da problemática, diversos modelos explicativos são observados, variando de acordo com valores pessoais, sociais, culturais, locais de inserção, dentre outros aspectos. Apesar de coexistirem, esses modelos nem sempre dialogam (PILLON; LUIS, 2004; SCHNEIDER, 2010). No entanto, em se tratando de um problema multifacetado e complexo, as tentativas de respostas devem ir numa direção de amplitude e abrangência, pautadas na problematização que vise potencializar as práticas profissionais e a qualidade de vida das pessoas (PILLON; LUIS, 2004).

A partir dessas constatações, os resultados das pesquisas analisadas apontam a predominância de visões morais, naturalizantes e pautadas pelo modelo biomédico dos profissionais de saúde sobre o uso de drogas, em oposição às perspectivas psicossociais ou socioculturais, ou mesmo biopsicossociais. Por mais que os estudos possuam uma diversidade metodológica (tamanho de amostra, métodos de coleta e análise etc.), implicando diferentes perspectivas no que concerne à capacidade de comparação e generalização de seus achados, observouse uma consonância dos artigos analisados em direção a esses aspectos. A literatura demonstra como visões negativas e estigmatizantes sobre o uso de drogas pelos profissionais de saúde influenciam suas atitudes, tornando-se obstáculos para: 1) a implementação de ações sobre o tema (AMARAL; RONZANI; SOUZAFORMIGONI 2010), 2) a qualidade das ações (AMARAL-SABADINI et al., 
2012), 3) a procura e adesão ao tratamento pelos usuários (FONTANELLA;

TURATO, 2002), dentre outros problemas em diversos âmbitos psicológicos, de saúde e sociais dos usuários (RONZANI; NOTO; SILVEIRA, 2014).

Ademais, coaduna-se com Traverso-Yepez (2001, p. 49), quando aponta que "[a]pesar de muito se falar do modelo biopsicossocial, observa-se que se continua privilegiando a etiologia biologicista, a concepção fragmentada de saúde, esquecendo-se a relevância dos aspectos sociais, psicológicos e ecológicos como mediadores dos processos saúde-doença”. Ou seja, a ideia do homem biopsicossocial e integral é pensada, ou ao menos externada, mas a prática é contraditória. Isso também não significa desconsiderar as contribuições e avanços no conhecimento provenientes da medicina, áreas afins e especialidades, como a biomedicina, neurociências, farmacologia, psiquiatria etc., mas compreendê-las por um prisma interdisciplinar necessário para a concepção do homem em sua integralidade biopsicossocial.

Nessa direção, considera-se importante que os determinantes sociais da saúde (DSS) relacionados ao uso de drogas sejam problematizados. Os DSS representam os diversos aspectos da realidade social (perpassando questôes intraindividuais, condições socioeconômicas e macroestruturais) e o quanto influenciam no processo saúde-doença (BUSS; FILHO, 2007). Assim como os aspectos biológicos e psicológicos, as condições de vida conformadas pela pobreza, relações hierárquicas de gênero, baixa escolaridade, desemprego, más condições de trabalho e moradia, dentre outros fatores, relacionam-se direta ou indiretamente ao uso de drogas e entre si (GALEA; VLAHOV, 2002; BASTOS; BERTONI, 2014).

Portanto, deve-se pensar no uso de drogas para além de uma doença meramente, ou uma prática aprioristicamente negativa ou danosa, mas como algo inerente à esfera humana, tendo seu nível de complexidade e consequências negativas influenciados pela relação que as pessoas estabelecem com as próprias substâncias, o que significa compreender seus aspectos físicos, psicológicos e também as dimensões da realidade em que são conformadas. Considera-se necessário debater a questão por um prisma que compreenda a dependência de drogas como uma condição crônica de saúde, o que não quer dizer reduzi-la aos componentes biológicos e/ou psicológicos, nem compreendê-la como uma mera patologia ou doença incapacitante a priori, levando a possíveis patologizações e 
culpabilizações do usuário (SCHNEIDER, 2010). Essa concepção de condição crônica indica a necessidade de se considerar um continuum de evolução/ gravidade, que pode ir do uso de baixo risco a diversos níveis de dependência, mas também os aspectos sociais relacionados e o cuidado, a partir de ações de promoção de saúde, prevenção e diversas modalidades de tratamento, reabilitação e (re)inserção social (RUSH, 2010).

Isso também não significa desconsiderar os possíveis prejuízos do uso de drogas, como apontam os estudos epidemiológicos (WHITEFORD et al., 2013), mas apenas entendê-los a partir de uma perspectiva realista e que ultrapasse a esfera individual embasada em subjetivismos e organicismos. Assim como todas as questōes referentes à vida, o uso de drogas não está descolado da realidade, inscrevendo-se no âmbito da sociedade vigente. Logo, não se considera oportuno compreender quaisquer atividades humanas, como o uso de drogas, sem inserilas no conjunto de relaçôes sociais sob as quais as pessoas se constituem.

Ressalta-se que o cenário encontrado é resultado de um processo sóciohistórico, em que a visão sobre o uso de drogas no Brasil foi (e ainda é) permeada prioritariamente pelos discursos da psiquiatria clássica e segurança pública, com vieses reducionistas, patologizantes e/ou criminalizatórios. Soma-se a isto a negligência estatal sobre a questão do uso de drogas, que favoreceu a inclusão de atores e organizaçôes da sociedade civil na abordagem da questão, trazendo consigo perspectivas religiosas de profunda conotação moral (ALVES, 2009; MACHADO; MIRANDA, 2007, MORAES, 2008). Tais perspectivas, em consonância com o imaginário social, corroboram uma visão negativa do usuário, seja pela via da fraqueza de caráter ou da irracionalização, ao possuir uma doença que age no sistema nervoso central e que inibe suas faculdades racionais/mentais.

Observou-se também uma predominância de estudos sobre enfermeiros e de publicações em revistas da Enfermagem, o que pode demonstrar a apropriação sobre o tema por determinados pesquisadores da área, mas também a necessidade de se repensar a atuação dessa categoria profissional frente à problemática (PILLON; LUIS, 2004), assim como outras (Psicologia, Medicina etc.). Fica para os estudos futuros a possibilidade de análises comparativas e integrativas, incorporando os diferentes saberes e categorias profissionais que perpassam a abordagem sobre a temática e possibilitando comparações, mas, ao mesmo tempo, visões multi ou interdisciplinares. 
Apesar da importância da APS na assistência aos usuários de drogas, estudos futuros também podem abarcar as recentes modificações nas políticas sobre drogas no Brasil, como a RAPS, seus dispositivos e níveis de atenção. Equipes de CAPSad, Unidades de Acolhimento, NASFs, consultórios na rua, comunidades terapêuticas, dentre outros, desempenham importante papel na rede de atenção aos usuários de drogas (BRASIL, 2011), devendo ter suas concepções englobadas de modo a compreender suas abordagens e potencializar o cuidado integral.

Uma das limitações do presente estudo é seu foco na concepção dos profissionais somente da saúde. Entender o uso de drogas como uma questão de saúde pública não significa relegá-lo somente ao setor saúde. Tendo em mente a complexidade da problemática e sua relação com os determinantes sociais, tem-se a necessidade de considerar outros setores, como o Sistema Único de Assistência Social (SUAS) e os dispositivos da segurança pública, seus serviços, profissionais e concepções na abordagem integral aos usuários de drogas (BRASIL, 2012).

Outro aspecto pertinente de discussão diz respeito à concentração de estudos na região Sudeste. Faz-se necessário realizar estudos com profissionais de diferentes contextos e localidades, abrangendo outras regióes, de modo a reconhecer se tais concepções são compartilhadas de forma horizontal no cenário nacional, e também estabelecer, através de comparações, possíveis relações entre aspectos socioculturais e as concepções existentes.

Por fim, os estudos apontam a necessidade de conscientização e sensibilização dos profissionais de saúde sobre o tema, em conjunto com maiores conhecimentos sobre o problema. Como principal mecanismo de reversão do cenário encontrado está a formação específica na área, seja na graduação ou com cursos técnicos, assim como no próprio processo de trabalho, de forma contínua (BARROS; PILLON 2007; VARGAS; LUÍS, 2008; SILVEIRA et al., 2009; MORETTIPIRES et al., 2010; 2011; SOARES et al., 2013; SILVA; PERES, 2014). Com relação a essa insuficiência de capacitação e formação na área, observa-se uma concordância com a literatura nacional (KANNO; BELLODI; TESS, 2012; VARGAS; DUARTE, 2011).

Como pode ser percebida, a questão vai além da mera insuficiência de conteúdo, sendo perpassada por valores e ideais compartilhados pela sociedade em geral. Sendo assim, deve-se pensar não somente na necessidade de capacitação, mas também nos pressupostos e metodologias que fundamentam esses processos 
formativos, indo além de aspectos tecnicistas e da mera transmissão de conteúdo, e abrindo espaço para a problematização da realidade, através de perspectivas participativas, horizontais e abertas às demandas das pessoas, dos cotidianos de vida e trabalho (COSTA et al., 2015).

As seguintes limitações fazem-se presentes no estudo: desconsideração de artigos indexados em outras bases de dados; terminologia utilizada a partir de dicionário de termos que facilita a sistematização, mas pode desconsiderar artigos e periódicos que não utilizem essa padronização; a não inclusão de dissertações, teses e relatos de experiência de difícil sistematização; as diferentes orientações epistemológicas, teóricas e metodológicas dos estudos, que, a depender da maneira como abordam seus objetos, podem direcionar as respostas aqui sinalizadas. Entretanto, acredita-se que essas limitações não invalidam os achados e a relevância da presente revisão.

\section{Considerações finais}

Ao abarcar as concepções dos profissionais de saúde sobre o uso de drogas, a presente revisão demonstra que os avanços nas legislações e políticas nacionais, por mais importantes que sejam, são limitados para modificar as visões sobre o tema. Contudo, através do conhecimento dessas concepções, pode-se iniciar o debate que vise considerar os atores que fazem parte desses contextos, entendendo essas concepções como resultados de processos históricos. Existe a tendência de se olhar para o profissional de saúde descolado da sociedade na qual ele se insere. Por mais que se espere que o processo de formação e o papel que ocupam tragam substratos importantes para uma visão mais integral e contextualizada sobre diversos temas, seus entendimentos sobre determinados assuntos são atravessados por valores societários, assim como qualquer outro sujeito.

Sendo assim, permanecem os desafios de reversão do cenário encontrado, em conjunto com algumas reflexões e possibilidades. A formação emerge como um caminho possível, mas com a necessidade de também ser problematizada, especialmente no que se refere a seus pressupostos e metodologias. Estudos e revisões futuras podem considerar as concepções dos próprios usuários de drogas, numa tentativa de se obter uma visão mais abrangente, buscando compreender como eles se percebem e quais são as reverberações das concepçôes dos diferentes grupos, setores e instituições sociais sobre eles. ${ }^{1}$ 


\section{Referências}

ALVES, V. S. Modelos de atenção à saúde de usuários de álcool e outras drogas: discursos políticos, saberes e práticas. Cad. Saúde Pública. Rio de Janeiro, v. 25, n. 11, p. 2309-2319, 2009. AMARAL, M. B.; RONZANI, T. M.; FORMIGONI, M. L. O. S. Process evaluation of the implementation of a screening and brief intervention program for alcohol risk in primary health care: An experience in Brazil. Drug Alcohol Rev, v. 29, p. 162-8, 2010.

AMARAL-SABADINI, M. B. et al. Is a patient's type of substance dependence (alcohol, drug or both) associated with the quality of primary care they receive? Qual PrimCare, v. 20, n. 6, p. 391-9, 2012.

BARDIN, L. Análise de conteúdo. Lisboa: Edições 70, 2010.

BARROS, M. A.; PILLON, S. C. Atitudes dos profissionais do Programa Saúde da Família diante do uso e abuso de drogas. Esc. Anna Nery. Rio de Janeiro, v. 11, n. 4, p. 655-2, 2007. BASTOS, F.; BERTONI, N. Pesquisa Nacional sobre o uso do crack: quem são os usuários de crack e/ou similares do Brasil? Quantos são nas capitais brasileiras? Rio de Janeiro: Editora ICICT/FIOCRUZ, 2014.

BRASIL. Ministério da Saúde. Portaria no 3.088, de 23 de dezembro de 2011. Institui a Rede de Atenção Psicossocial para pessoas com sofrimento ou transtorno mental e com necessidades decorrentes do uso de crack, álcool e outras drogas, no âmbito do Sistema Único de Saúde. Brasília: Diário Oficial da União, 2011.

. Tribunal de Contas da União. Sistema nacional de políticas públicas sobre drogas. Brasília: Tribunal de Contas da União, 2012.

BUSS, P. M.; FILHO, A. P. A Saúde e seus Determinantes Sociais. Physis: Revista de Saúde Coletiva. Rio de Janeiro, v. 17, n. 1, p. 77-93, 2007.

CASTANHO, A. R.; ARAÚJO, L. F. Álcool e agentes comunitários de saúde: um estudo das representações sociais. Psico-USF. Itatiba, v. 11, n. 1, p. 85-94, 2006.

COSTA, P. H. A. et al. Capacitação em álcool e outras drogas para profissionais da saúde e assistência social: Relato de experiência. Interface. Botucatu, v. 19, n. 53, p. 395-404, 2015. FONTANELLA, B. J. B.; TURATO, E. R. Barreiras na relação clínico-paciente em dependentes de substâncias psicoativas procurando tratamento. Rev. Saúde Públ., São Paulo, v. 36, n. 4, p. 439-47, 2002.

GALEA, S.; VLAHOV, D. Social determinants and the health of drug users: socioeconomic status, homelessness, and incarceration. Public Health Rep., v. 117, supl. 1, p. 135-45, 2002. GOMIDE, H. P. et al. Estereótipos dos profissionais de saúde em relação a alcoolistas em Juiz de Fora-MG, Brasil. Psicologia: Teoria e Prática. São Paulo, v. 12, n. 1, p. 171-80, 2010. JAPIASSÚ, H.; MARCONDES, D. Dicionário básico de filosofia. Rio de Janeiro, Jorge Zahar, 2001. 
KANNO, N. P.; BELLODI, P. L.; TESS, B. H. Profissionais da Estratégia Saúde da Família diante de demandas médico-sociais: dificuldades e estratégias de enfrentamento. Saude soc. São Paulo, v. 21, n. 4, p. 884-894, 2012.

LIMA, M. et al. Invisibilidade do uso de drogas e a assistência de profissionais dos serviços de Aids. Rev. Saúde Públ. São Paulo, n. 41, supl. 2, p. 6-13, 2007.

MACHADO, A. R.; MIRANDA, P. S. C. Fragmentos da história da atenção à saúde para usuários de álcool e outras drogas no Brasil. Hist. cienc. saude-Manguinhos. Rio de Janeiro, v. 14, n. 3, p. 801-21, 2007.

MARTINS, E. R. C. et al. Concepçôes do trabalhador de enfermagem sobre drogas: a visibilidade dos riscos. Rev Enferm UERJ. Rio de Janeiro, v. 17, n. 3, p. 368-72, 2009.

MORAES, M. Integral healthcare model for treating problems caused by alcohol and other drugs: perceptions of users, their companions and practitioners. Ciên. saúde colet. Rio de Janeiro, v. 13 n. 1, p. 121-133, 2008.

MORETTI-PIRES, R. O. et al. Enfermeiro de Saúde da Família na Amazônia: conceitos e manejo na temática do uso de álcool. Rev Esc Enferm USP. São Paulo, v. 45, n. 4, p. 926-32, 2011.

MORETTI-PIRES, R. O.; LIMA, L. A. M.; KATSURAYAMA, M. A formação dos médicos de saúde da família no interior da Amazônia sobre a problemática do abuso de álcool. Rev. bras. prom. saúde. Fortaleza, v. 23, n. 1, p. 56-62, 2010.

MOUTINHO, E. C. V. S.; LOPES, G. T. Enfermeiro do Programa de Saúde da Família: conceitos e crenças sobre drogas e modelos teóricos explicativos. Rev Enferm UERJ. Rio de Janeiro, v. 16, n. 1, p. 51-7, 2008.

OLIVEIRA, J. F. et al. Representações sociais de profissionais de saúde sobre o consumo de drogas: um olhar numa perspectiva de gênero. Ciênc Saude Coletiva Rio de Janeiro, v. 11, n. 2, p. 473-81, 2006.

OliveiRA, J. F.; McCAlluM, C. A.; COSTA, H. O. G. Representações sociais de Agentes Comunitários de Saúde acerca do consumo de drogas. Rev. esc. enferm. USP. São Paulo, v. 44, n., p. 611-18, 2010.

PAIVA, F. S. et al. A percepção profissional e comunitária sobre a reinserção social dos usuários de drogas. Psicol. Soc. Belo Horizonte, v. 26, n. 3, p. 696-706, 2014.

PILLON, S. C.; LUIS, M. A. V. Modelos explicativos para o uso de álcool e drogas e a prática da enfermagem. Rev. Latino-Am. Enfermagem. Ribeirão Preto, v. 12, n. 4, p. 676-82, 2004.

PRATES, J. G. et al. A concepção dos enfermeiros de serviços de urgência e emergência sobre o processo saúde-doença na assistência aos usuários de substâncias psicoativas. Saúde Debate. Rio de Janeiro, v. 38, n. 101, p. 318-27, 2014. 
REZENDE, M. M. Uma provável caracterização do profissional de saúde mental que atende dependentes de drogas no Vale do Paraíba. Rev. latinoam. psicopatol. fundam. São Paulo, v. 6, n. 1, p. 100-8, 2003.

RONZANI, T. M.; HIGGINS-BIDDLE, J.; FURTADO, E. F. Stigmatization of alcohol and other drug user by primary care providers in Southeast Brazil. Social Science \& Medicine, v. 69, n. 7, p. 1080-84, 2009.

RONZANI, T. M.; NOTO, A. R.; SILVEIRA, P. S. Reduzindo o estigma entre usuários de drogas. Guia para profissionais e gestores. Juiz de Fora: Editora UFJF, 2014.

RUSH, B. Tiered frameworks for planning substance use service delivery systems: origins and key principles. Nordic Studies on Alcohol and Drugs, v. 27, p. 617-636, 2010.

SCHNEIDER, D. R. Horizonte de racionalidade acerca da dependência de drogas nos serviços de saúde: implicações para o tratamento. Ciênc Saude Coletiva. Rio de Janeiro, v. 15, n. 3, p. 687-98, 2010.

SILVA, W. R.; PERES, R. S. Concepções sobre álcool e outras drogas na atenção básica: o pacto denegativo dos profissionais de saúde. Psicol., Ciênc. Prof. Brasília, v. 34, n. 2, p. 474-87, 2014.

SILVEIRA, P. S.; MARTINS, L. F.; RONZANI, T. M. Moralização sobre o uso de álcool entre agentes comunitários de saúde. Psicologia: Teoria e Prática. São Paulo, v. 11, p. $62-75,2009$.

SOARES, J.; VARGAS, D.; FORMIGONI, M. L. O. S. Atitudes e conhecimentos de enfermeiros frente ao álcool e problemas associados: impacto de uma intervenção educativa. Rev Esc Enferm USP. São Paulo, v. 47, n. 5, p. 1172-9, 2013.

SPRICIGO, J. S.; ALENCASTRE, M. B. O enfermeiro de unidade básica de saúde e o usuário de drogas: um estudo em Biguaçú-SC. Rev. Latino-Am. Enfermagem. Ribeirão Preto, v. 12, n. esp., p. 427-32, 2004.

TRAVERSO-YÉPEZ, M. A interface psicologia social e saúde: perspectivas e desafios. Psicol. estud. Maringá, n. 6, v. 2, p. 49-56, 2001.

VARGAS, D.; DUARTE, F. A. B. Enfermeiros dos centros de atenção psicossocial em álcool e drogas (Caps ad): a formação e a busca pelo conhecimento específico da área. Texto contexto - enferm. Florianópolis, v. 20, n. 1, p. 119-26, 2011.

VARGAS, D.; LABATE, R. C. Atitudes de enfermeiros de hospital geral frente ao uso do álcool e alcoolismo. Rev Bras Enferm. Brasília, v. 59, n. 1, p. 47-51, 2006.

VARGAS, D.; LUIS, M. A. V. Alcohol, alcoholism and alcohol addicts: conceptions and attitudes of nurses from district basic health centers. Rev. Latino-Am. Enfermagem. Ribeirão Preto, v. 16, n. esp., p. 543-50, 2008. 
WHITEFORD, H. A. et al. Global burden of disease attributable to mental and substance use disorders: findings from the Global Burden of Disease Study 2010. The Lancet, v. 382, p. 1575-86, 2013.

\section{Nota}

${ }^{1}$ P. H. A. da Costa participou da concepção e desenho do estudo, coleta e análise do material, escrita e revisão do manuscrito. F. S. de Paiva participou da concepção e desenho do estudo, coleta e análise do material, escrita e revisão do manuscrito. 
Literature review on the conceptions of health professionals on the use of drugs in Brazil: biomedical model, naturalizations and moralism

This study aimed to understand the conceptions of Brazilian health professionals on drug use/abuse. It is an academic literature review in the following databases: MEDLINE, LILACS, IBECS and Scielo. The final sample consisted of 22 articles, with the results pointing to a predominance of moral and naturalized conceptions about drug use, guided by biomedical model rather than psychosocial, socio-cultural or even biopsychosocial perspectives. In the case of a multi-faceted and complex problem, answers' attempts should go in the direction of breadth and comprehensiveness, thinking drug use beyond a mere disease, or necessarily a bad thing, but understanding the physical, psychological and social aspects that pervade it. For the reversal of this scenario, it is necessary training/capacity building in the area, but reflecting the premises and methodologies that underlie these training processes.

Key words: substance-related disorders; alcohol-related disorders; health personnel; health manpower; review. 